\title{
Impactos das assistências obstétricas e dos tipos de partos: experiências de primíparas
}

\section{Obstetricians assistances impacts and deliveries methods: primiparous experiences}

DOI: $10.46814 / 1 a j d v 3 n 5-034$

Recebimento dos originais: 01/05/2021

Aceitação para publicação: 31/06/2021

\section{Prof. Dr. Joe Luiz Vieira Garcia Novo}

Professor Titular do Departamento de Reprodução Humana e Infância. Faculdade de Ciências Médicas e Saúde - PUC/SP

Endereço: Rua Manoel Ferreira Leão, 67 ap 21

Vila Leão - Sorocaba-SP

CEP 18040-410

E-mail: joeluiznovo@ hotmail.com

Ana Alice Amaral de Oliveira

Ex - Acadêmica do Curso de Medicina. Faculdade de Ciências Médicas e Saúde - PUC/SP

\section{Lízia Baruque Baylão}

Ex - Acadêmica do Curso de Medicina. Faculdade de Ciências Médicas e Saúde - PUC/SP

Paula Fenili

Ex - Acadêmica do Curso de Medicina. Faculdade de Ciências Médicas e Saúde - PUC/SP

Prof. Dr. Neil Ferreira Novo

Ex - Professor Titular do Departamento de Morfologia. Faculdade de Ciências Médicas e Saúde PUC/SP

\section{RESUMO}

Objetivos: conhecer impactos e experiências em partos (vaginais e cesáreos) de primíparas. Métodos: estudadas 306 puérperas de termo (SUS), impactos e experiências nos partos esperados e ocorridos. Utilizou-se escala de valores para respostas psicométricas "em nenhum momento", "alguns momentos", "frequente", "maior parte do tempo" e "tempo todo". A cada termo indicativo da frequência de ocorrência foi atribuído número de 1 a 5 . O questionário possuía duas partes: na primeira indicavam aspectos positivos quanto maior a frequência de ocorrência e na segunda, as experiências negativas, onde perguntas citadas exibiam exatamente as respostas inversas. Resultados: para a via de parto preferencial no acompanhamento pré-natal e o parto realizado: $101(53,72 \%)$ ficaram satisfeitas com seu parto vaginal ocorrido, enquanto $88(74,58 \%)$ dos partos cesáreas, desaprovaram a cesárea resultante ( $\mathrm{p}<0$, 0001). Proporções maiores, positivas e significantes foram após o parto vaginal, por ser opção pessoal, rápida recuperação, melhor sensação com recém-nascido, receio de acidentes anestésicos. Provável incapacidade para em sua execução, o tempo e a dor intraparto, geraram impactos negativos significantes $(\mathrm{p}<0,0001)$. Estatisticamente significante, menor em preferência, a cesárea surgiu favoravelmente $(\mathrm{p}<0,0001)$ contra a dor intraparto e rapidez resolutiva; porém, foi desaprovada pela possibilidade de acidente anestésico ( $\mathrm{p}<0,0001)$. Conclusões: Predominaram favoravelmente experiências positivas para parto vaginal, com significância estatística. Impactos negativos para eles 
foram o tempo, sensação dolorosa e provável incapacidade para a execução partal. As soluções sugeridas para minimizá-las: atenções assistenciais adequadas devolvendo a autoconfiança às pacientes, além da utilização da analgesia intraparto. Apesar de significantemente menor em preferência, o parto cesáreo foi avaliado positivamente pelo receio da experiência dolorosa da parturição, e por sua rapidez resolutiva. A cesárea. teve significante e negativamente contra si, a possibilidade de acidente anestésico.

Descritores: assistência obstétrica, via de parto, parto normal, cesárea, experiências de primíparas.

\begin{abstract}
Objectives: to know impacts and experiences in births (vaginalists and cesareans) of primiparous. Methods: 306 mothers at term (SUS) were studied, impacts and experiences in expected and actual deliveries. A scale of values was used for psychometric responses "at no time", "some moments", "frequent", "most of the time" and "all time". Each term indicating the frequency of occurrence was assigned a number from 1 to 5. The questionnaire had two parts: first indicated positive aspects, the higher the frequency of occurrence and the second, negative experiences, where the questions mentioned showed exactly the opposite answers. Results: for the preferred mode of delivery in prenatal care and delivery: $101(53.72 \%)$ were satisfied with their vaginal delivery, while $88(74.58 \%)$ of the cesarean deliveries disapproved the resulting cesareans ( $p<0.0001$ ). Larger, positive, and significant proportions were after vaginal delivery, as it is a personal option, quick recovery, better feeling with the newborn, fear of anesthetic accidents. Probable inability to perform, time and intrapartum pain, generated significant, negative impacts $(\mathrm{p}<0,0001)$. Statistic significant, lower in preference, the cesarean appeared favorably $(\mathrm{p}<0.0001)$ against intrapartum pain and resolving speed; however, it was disapproved due to the possibility of an anesthetic accident $(\mathrm{p}<0,0001)$. Conclusions: Positive experiences for vaginal delivery predominated, with statistical significance. Negative impacts for them were timing, painful sensation, and likely inability to perform birth. Suggested solutions to minimize them: adequate care assistance restoring self-confidence to patients, in addition to the use of intrapartum analgesia. Despite being significantly lower in preference, cesarean delivery was evaluated positively for fear of the painful experience of parturition, and for its rapid resolution. The cesarean. had significantly and negatively against itself, the possibility of an anesthetic accident.
\end{abstract}

Keywords: obstetric assistance, way of delivery, normal delivery, cesarean section, primigravid experience.

\title{
1 INTRODUÇÃO
}

O sexo faz parte do cotidiano feminino englobando o seu prazer humano, que pode ser associado à capacidade reprodutiva, cabendo à mulher conhecer e participar de sua própria sexualidade e de seu planejamento familiar. ${ }^{1}$

Porém, o alto nível de desinformação, ainda, presente atualmente, descrito em literatura nacional e internacional, pode refletir as dificuldades em abordagem do corpo e à sexualidade feminina, pela ausência de programas educativos em escolas e/ou serviços de saúde. ${ }^{2,3}$

O feminismo vem obtendo importantes conquistas em todo o mundo, inclusive no Brasil, mas, observa-se que a maioria das iniciativas relacionadas à saúde das mulheres, vem-se caracterizando em manipular seus corpos, suas vidas, visando outros objetivos e não propriamente o seu bem-estar. 
Estes programas idealmente expropriam a autodeterminação da mulher, como atestam os altos índices de morbiletalidade materna e perinatal, prática abusiva de partos cesáreos, prevalência de esterilizações e procedimentos contraceptivos inadequados. ${ }^{4}$

Desta forma, o processo natural da evolução de um trabalho de parto, para a mulher com organismo fisiologicamente preparado, para a não ocorrência de complicações, torna-se objeto de rotinas, despersonalizado, nos quais a parturiente, em certas ocasiões, não recebe o tratamento humanístico a que tem direito. ${ }^{4,5}$

No momento da parturição, a assistência hospitalar tem sido objeto de medicalização em larga escala, a mulher sofre durante este evento perdendo a sua própria autonomia em relação ao seu corpo. 6

A possibilidade da vivência de uma gravidez é, sem dúvida, momento excepcional na vida de, pelo menos, na maioria das mulheres. As modificações gravídicas geram alterações físicas e/ou psicológicas, as quais, associadas às orientações leigas ou profissionais, podem inserir impactos favoráveis ou não, dependentes das qualidades fornecidas. ${ }^{5,7}$

A importância das informações transmitidas durante a evolução da gravidez, através de profissionais qualificados na assistência pré-natal, será relevante orientando e informando a opinião das mulheres, quanto às possibilidades de vivência e escolha da futura via de parto. ${ }^{8}$

Será fundamental a toda mulher ter garantias dos avanços científicos, que a assistência hospitalar irá propiciar ao trabalho de parto, mas, também, entenda-se que este momento seja definido, permitindo e estimulando o exercício da cidadania feminina, trazendo e resgatando a mulher em relação a seu parto. ${ }^{6}$

Este estudo teve como objetivo conhecer na atualidade, os impactos das assistências obstétricas e dos partos ocorridos (vaginais e cesáreos) em pacientes primíparas, assistidas em hospital universitário de assistência secundária.

Visou a probabilidade de, se necessário, melhorar a qualidade de relacionamento entre médicos e pacientes, da assistência obstétrica, assim como, de uma maior autonomia e compreensão da mulher, em relação ao seu parto.

\section{METODOLOGIA}

Incluíram-se 306 puérperas do Sistema Único de Saúde (SUS), primíparas de termo, sem razão médica que exigisse cesárea eletiva quando de sua internação, atendidas na maternidade do Hospital Santa Lucinda de Sorocaba-SP (HSL), em pesquisa prospectiva. As pacientes avaliadas concordaram e assinaram afirmativamente o Termo de Conhecimento Livre e Esclarecido, para participarem desta pesquisa. 
O projeto iniciou-se após a submissão e aprovação do Comitê de Ética em Pesquisa (CEP) da Faculdade de Ciências Médicas e da Saúde (FCMS/PUC-SP). Respeitaram-se as normas da Declaração de Helsinque ${ }^{9}$ e da resolução $n^{\circ} 466 / 2012$ do Conselho Nacional de Saúde para pesquisa em seres humanos. ${ }^{10}$

Foram estudadas as expectativas das pacientes sobre as assistências, e os tipos de partos esperados e ocorridos, considerando-se a via vaginal ou cesárea. Como variáveis maternas de controle consideraram-se: fatores epidemiológicos, socioeconômicos e assistência pré-natal prévia.

As pacientes que foram incluídas no momento de suas avaliações eram puérperas, no mínimo, com seis horas de pós-parto, em condições de responder à entrevista de forma consentida e informada. 11

A escolha de pacientes em primeira gestação, para a avaliação dos procedimentos assistenciais obstétricos atuais, justificou-se pelo fato de que é inegável a possibilidade de influência de partos anteriores, sobre os prováveis resultados a serem estudados nesta pesquisa. ${ }^{12}$

As questões (experiências, satisfação, dor ou qualquer outro relato) pesquisadas, que poderiam se relacionar com sua ocorrência e o tipo de parto executado, foram esclarecidas a cada puérpera, propondo-se obter a sua resposta individual e verbal na pesquisa. Utilizou-se uma escala de valores para as respostas psicométricas de: "em nenhum momento", "alguns momentos", "frequente", "maior parte do tempo" e "tempo todo". A cada um dos termos indicativos da frequência de ocorrência foi atribuído um número de 1 a 5 . O questionário possuía duas partes. Na primeira parte os questionamentos indicavam aspectos positivos, quanto maior a frequência de ocorrência. Assim sendo, na primeira parte o termo "em nenhum momento" é representado por 1, "alguns momentos" são representados por 2, "frequente" é representado por 3, "maior parte do tempo" é representado por $4 \mathrm{e}$ "tempo todo" é representado por 5. Na segunda parte, pautada por experiências negativas, as perguntas citadas exibiam exatamente as respostas inversas: "em nenhum momento" é 5, "alguns momentos" será 4 , e assim até o valor $1 .^{13}$

Para a análise estatística o teste do qui-quadrado foi utilizado, com o objetivo de comparar os fatores considerados. Em todos os testes fixou-se o nível de significância em 0.05 ou $5 \%$. ${ }^{14,15} \mathrm{O}$ teste da razão de verossimilhança foi aplicado, com o intuito de ser verificado possíveis diferenças entre as categorias de parto preferencial (normal, cesárea e/ou indiferente). ${ }^{16}$

\section{RESULTADOS}

Analisaram-se 306 pacientes. A utilização do teste da razão da verossimilhança para as variáveis de interesse informadas, revelou não haver estatística significante em nenhuma das diferenças, encontradas no tipo de parto de preferência inicial delas, em relação a qualquer um dos 
fatores epidemiológicos e socioeconômicos descritos (dispensando a apresentação de tabela demonstrativa).

Em relação à via de parto preferencial durante o acompanhamento pré-natal e o parto realizado: $101(53,72 \%)$ ficaram satisfeitas com o parto vaginal ocorrido, enquanto $88(74,58 \%)$ dos partos cesáreas, não aprovaram a via de parto cirúrgico resultante. Esta diferença foi estatisticamente significante $(\mathrm{p}<0,0001)$ (Tabela 1$)$.

TABELA 1: Aplicação do Teste de Qui-quadrado, com o intuito de verificarmos possíveis diferenças entre as categorias de 'parto preferencial', para a variável de interesse 'parto'.

\begin{tabular}{cccc}
\hline \multirow{2}{*}{ Parto } & \multicolumn{2}{c}{ Parto preferencial } & \multirow{2}{*}{ Total } \\
\cline { 2 - 3 } & Normal & Cesárea & \\
\hline realizados & 101 & 30 & 131 \\
\hline não realizados & 87 & 88 & 175 \\
\hline Total & 188 & 118 & 306 \\
\hline \multicolumn{3}{c}{$\mathrm{p}<0,001$}
\end{tabular}

Analisando-se os motivos de escolha para determinado tipo de parto ao adentrarem na maternidade, havia significante preferência pela via vaginal, manifestada por opção pessoal ao futuro parto, recuperação pós-parto mais rápida e receio de acidentes anestésicos $(\mathrm{p}<0,0001)$. A favor do parto cesáreo, seria o receio de provável experiência dolorosa do trabalho de parto $(\mathrm{p}<0,0001)$ (Tabela 2).

TABELA 2: $\quad$ Motivo Escolha antes do Parto: Parto Normal (PN) X Parto Cesárea (PC)

\begin{tabular}{|l|c|c|c|c|}
\hline \multicolumn{5}{|c|}{ Medo Anestesia } \\
\hline & Presente & Ausente & Total pref. & $\%$ Presença \\
\hline PN & 65 & 123 & 188 & 26 \\
\hline PC & 6 & 112 & 118 & 10,7 \\
\hline TOTAL & 71 & 235 & 306 & 23,2026144 \\
\hline \multicolumn{5}{|c|}{$\mathrm{p}<0,0001$} \\
\hline
\end{tabular}

Medo da dor

\begin{tabular}{|l|c|c|c|c|}
\hline & Presente & Ausente & Total pref. & $\%$ Presença \\
\hline PN & 32 & 156 & 188 & 12,8 \\
\hline PC & 44 & 74 & 118 & 78,6 \\
\hline TOTAL & 76 & 230 & 306 & 24,836601 \\
\hline \multicolumn{5}{|c|}{$\mathrm{p}<0,0001$} \\
\hline
\end{tabular}

\begin{tabular}{|l|c|c|c|c|}
\hline \multicolumn{5}{|c|}{ Posição do nenê } \\
\hline & Presente & Ausente & Total pref & \% Presença \\
\hline PN & 12 & 176 & 188 & 4,8 \\
\hline PC & 3 & 115 & 118 & 5,4 \\
\hline TOTAL & 15 & 291 & 306 & 4,90196078 \\
\hline \multicolumn{5}{|c|}{ p<0,0001 } \\
\hline
\end{tabular}

\begin{tabular}{|l|c|c|c|c|}
\hline \multicolumn{5}{|c|}{ Opção Pessoal } \\
\hline & Presente & Ausente & Total pref. & $\%$ Presença \\
\hline PN & 124 & 64 & 188 & 49,6 \\
\hline PC & 10 & 108 & 118 & 17,9 \\
\hline TOTAL & 134 & 172 & 306 & 43,79085 \\
\hline \multicolumn{5}{|c|}{$\mathrm{p}<0,0001$} \\
\hline
\end{tabular}




\begin{tabular}{|l|c|c|c|c|}
\hline \multicolumn{5}{|c|}{ Recuperação pós-parto } \\
\hline & Presente & Ausente & Total pref & $\%$ Presença \\
\hline PN & 73 & 115 & 188 & 29 \\
\hline PC & 7 & 111 & 118 & 12,5 \\
\hline TOTAL & 80 & 226 & 306 & 26,1437908 \\
\hline \multicolumn{5}{|c|}{$\mathrm{p}<0,0001$} \\
\hline
\end{tabular}

\begin{tabular}{|l|c|c|c|c|}
\hline \multicolumn{5}{|c|}{ Custo } \\
\hline & Presente & Ausente & Total pref. & \% Presença \\
\hline PN & 7 & 181 & 188 & 2,8 \\
\hline PC & 0 & 118 & 118 & 0 \\
\hline TOTAL & 7 & 299 & 306 & 2,2875817 \\
\hline \multicolumn{5}{|c|}{$\mathrm{p}<0,0001$} \\
\hline
\end{tabular}

Após a ocorrência do parto as experiências positivas estatisticamente significantes para o parto normal, foram: a emoção de poder ter tido parto vaginal que atendeu às expectativas prévias, a sensação de melhor encontro com seu recém-nascido, a qualidade dos cuidados da enfermagem e dos médicos assistentes ( $\mathrm{p}<0,0001)$. Em relação ao parto cesáreo foi significante apenas a rapidez do mesmo, que lhes chamou a atenção ( $\mathrm{p}<0,0001)$ (Tabela 3).

TABELA 3: $\quad$ Experiências Positivas - Parto Normal (PN) X Parto Cesárea (PC)

\begin{tabular}{|l|c|c|c|c|}
\hline \multicolumn{5}{|c|}{ Emoção } \\
\hline & Presente & Ausente & Total & $\%$ Presença \\
\hline PN & 95 & 93 & 188 & 50,5 \\
\hline PC & 33 & 85 & 118 & 28 \\
\hline TOTAL & 128 & 178 & 306 & 41,83006536 \\
\hline \multicolumn{5}{|c|}{$\mathrm{p}<0,0001$} \\
\hline
\end{tabular}

\begin{tabular}{|l|c|c|c|c|}
\hline \multicolumn{5}{|c|}{ Encontro com filho } \\
\hline & Presente & Ausente & Total & $\%$ Presença \\
\hline PN & 145 & 43 & 188 & 77,1 \\
\hline PC & 57 & 61 & 118 & 48,3 \\
\hline TOTAL & 202 & 104 & 306 & 66,0130719 \\
\hline \multicolumn{5}{|c|}{$\mathrm{p}<0,0001$} \\
\hline
\end{tabular}

\begin{tabular}{|l|c|c|c|c|}
\hline \multicolumn{5}{|c|}{ Rapidez } \\
\hline & Presente & Ausente & Total & \% Presença \\
\hline PN & 53 & 135 & 188 & 28,2 \\
\hline PC & 50 & 68 & 118 & 42,4 \\
\hline TOTAL & 103 & 203 & 306 & 33,66013072 \\
\hline \multicolumn{7}{|c|}{ p $<0,0001$} \\
\hline
\end{tabular}

\begin{tabular}{|l|c|c|c|c|}
\hline \multicolumn{5}{|c|}{ Atendeu as expectativas } \\
\hline & Presente & Ausente & Total & \% Presença \\
\hline PN & 92 & 96 & 188 & 49 \\
\hline PC & 29 & 89 & 118 & 24,6 \\
\hline TOTAL & 121 & 185 & 306 & 39,5424837 \\
\hline \multicolumn{5}{|c|}{$\mathrm{p}<0,0001$} \\
\hline
\end{tabular}

\begin{tabular}{|l|c|c|c|c|}
\hline \multicolumn{5}{|c|}{ Enfermagem } \\
\hline & Presente & Ausente & Total & $\%$ Presença \\
\hline PN & 133 & 55 & 188 & 70,7 \\
\hline PC & 41 & 77 & 118 & 34,7 \\
\hline TOTAL & 174 & 132 & 306 & 56,8627451 \\
\hline \multicolumn{7}{|c|}{ p $<0,0001$} \\
\hline
\end{tabular}

\begin{tabular}{|l|c|c|c|c|}
\hline \multicolumn{5}{|c|}{ Qualidade cuidados prestados } \\
\hline & Presente & Ausente & Total & $\%$ Presença \\
\hline PN & 122 & 66 & 188 & 64,9 \\
\hline PC & 52 & 66 & 118 & 44,1 \\
\hline TOTAL & 174 & 132 & 306 & 56,8627451 \\
\hline \multicolumn{5}{|c|}{$\mathrm{p}<0,0001$} \\
\hline
\end{tabular}

\begin{tabular}{|l|c|c|c|c|}
\hline \multicolumn{5}{|c|}{ Médicos } \\
\hline & Presente & Ausente & Total & \% Presença \\
\hline PN & 126 & 62 & 188 & 67 \\
\hline PC & 52 & 66 & 118 & 44,1 \\
\hline TOTAL & 178 & 128 & 306 & 58,16993464
\end{tabular}


Em relação às experiências negativas para o parto vaginal, houve significância estatística para a sensação de desconforto doloroso durante a parturição, o tempo necessário para a sua duração e de provável incapacidade para a execução do mesmo $(\mathrm{p}<0,0001)$. O parto cesáreo teve contra si negativamente o receio de acidente anestésico ( $<<0,0001)$ (Tabela 4).

TABELA 4: $\quad$ Experiências Negativas - Parto Normal (PN) X Parto Cesárea (PC)

\begin{tabular}{|l|c|c|c|c|}
\hline \multicolumn{5}{|c|}{ Parto Normal X Parto Cesárea EP } \\
\hline & Presença & $\begin{array}{c}\text { Ausênci } \\
\text { a }\end{array}$ & Total & \% Presença \\
\hline PN & 125 & 63 & 188 & 66.5 \\
\hline PC & 26 & 92 & 118 & 22.0 \\
\hline $\begin{array}{l}\text { TOTA } \\
\text { L }\end{array}$ & 151 & 155 & 306 & 49,34640523 \\
\hline
\end{tabular}

\begin{tabular}{|l|c|c|c|c|}
\hline \multicolumn{5}{|c|}{ Parto Normal X Parto Cesárea EP } \\
\hline & Presença & Ausência & Total & \% Presença \\
\hline PN & 9 & 179 & 188 & 4.8 \\
\hline PC & 8 & 110 & 118 & 6.8 \\
\hline $\begin{array}{l}\text { TOTA } \\
\text { L }\end{array}$ & 17 & 289 & 306 & 5,55555556 \\
\hline \multicolumn{5}{|c|}{ p <0,0001 } \\
\hline
\end{tabular}

\begin{tabular}{|l|c|c|c|c|}
\hline \multicolumn{5}{|c|}{ Parto Normal X Parto Cesárea EP } \\
\hline & Presença & $\begin{array}{c}\text { Ausênci } \\
\text { a }\end{array}$ & Total & \% Presença \\
\hline PN & 46 & 142 & 188 & 24.5 \\
\hline PC & 15 & 103 & 118 & 12.7 \\
\hline $\begin{array}{l}\text { TOTA } \\
\text { L }\end{array}$ & 61 & 245 & 306 & 19,93464052 \\
\hline \multicolumn{7}{|c|}{$\mathrm{p}<0,0001$} \\
\hline
\end{tabular}

\begin{tabular}{|l|c|c|c|c|}
\hline \multicolumn{5}{|c|}{ Parto Normal X Parto Cesárea EP } \\
\hline & Presença & Ausência & Total & $\%$ Presença \\
\hline PN & 2 & 186 & 188 & 1.1 \\
\hline PC & 1 & 117 & 118 & 0.8 \\
\hline $\begin{array}{l}\text { TOTA } \\
\text { L }\end{array}$ & 3 & 303 & 306 & 0,98039216 \\
\hline \multicolumn{7}{|c|}{ p<0,0001 } \\
\hline
\end{tabular}

\begin{tabular}{|l|c|c|c|c|}
\hline \multicolumn{5}{|c|}{ Parto Normal X Parto Cesárea EP } \\
\hline & Presença & $\begin{array}{c}\text { Ausênci } \\
\text { a }\end{array}$ & Total & \% Presença \\
\hline PN & 15 & 173 & 188 & 8 \\
\hline PC & 2 & 116 & 118 & 1.7 \\
\hline $\begin{array}{l}\text { TOTA } \\
\text { L }\end{array}$ & 17 & 289 & 306 & 5,555555556 \\
\hline \multicolumn{7}{|c|}{$\mathrm{p}<0,0001$} \\
\hline
\end{tabular}

\begin{tabular}{|l|c|c|c|c|}
\hline \multicolumn{5}{|c|}{ Parto Normal X Parto Cesárea EP } \\
\hline & Qualidade Cuidados Prestados \\
\hline & Presença & Ausência & Total & \% Presença \\
\hline PN & 0 & 188 & 188 & 0 \\
\hline PC & 2 & 116 & 118 & 1.7 \\
\hline $\begin{array}{l}\text { TOTA } \\
\text { L }\end{array}$ & 2 & 304 & 306 & 0,65359477 \\
\hline \multicolumn{5}{|c|}{$\mathrm{p}<0,0001$} \\
\hline
\end{tabular}

\begin{tabular}{|l|c|c|c|c|}
\hline \multicolumn{5}{|c|}{ Parto Normal X Parto Cesárea EP } \\
\hline & Presença & $\begin{array}{c}\text { Ausênci } \\
\text { a }\end{array}$ & Total & \% Presença \\
\hline PN & 8 & 180 & 188 & 4.3 \\
\hline PC & 37 & 81 & 118 & 31.4 \\
\hline
\end{tabular}

\begin{tabular}{|l|c|c|c|c|}
\hline \multicolumn{5}{|c|}{ Parto Normal X Parto Cesárea EP } \\
\hline & Atendeu às Expectativas \\
\hline & Presença & Ausência & Total & \% Presença \\
\hline PN & 2 & 186 & 188 & 1.1 \\
\hline PC & 2 & 116 & 118 & 1.7 \\
\hline
\end{tabular}




\begin{tabular}{|c|c|c|c|c|c|c|c|c|c|}
\hline $\begin{array}{l}\text { TOTA } \\
\text { L }\end{array}$ & 45 & 261 & 306 & 14,70588235 & $\begin{array}{l}\text { TOTA } \\
\text { L }\end{array}$ & 4 & 302 & 306 & 1,30718954 \\
\hline \multicolumn{5}{|c|}{$\mathrm{p}<0,0001$} & \multicolumn{5}{|c|}{$\mathrm{p}<0,0001$} \\
\hline
\end{tabular}

\begin{tabular}{|l|c|c|c|c|}
\hline \multicolumn{5}{|c|}{ Parto Normal X Parto Cesárea EP } \\
\hline & Presença & $\begin{array}{c}\text { Ausênci } \\
\text { a }\end{array}$ & Total & \% Presença \\
\hline PN & 3 & 185 & 188 & 1.6 \\
\hline PC & 2 & 116 & 118 & 1.7 \\
\hline $\begin{array}{l}\text { TOTA } \\
\text { L }\end{array}$ & 5 & 301 & 306 & 1,633986928 \\
\hline \multicolumn{7}{|c|}{$\mathrm{p}<0,0001$} \\
\hline
\end{tabular}

\section{DISCUSSÃO}

O Ministério da Saúde (MS) compreendeu que a inconsistente assistência obstétrica em nosso país, estava entrelaçada com a não percepção do reconhecimento da mulher como sujeito, além do total desconhecimento de seus direitos reprodutivos. Tentou resgatar estas deficiências, estabelecendo o Programa de Humanização no Pré-natal e Nascimento (PHPN). Esta cartilha procurava incentivar e concentrar os esforços nas atenções humanísticas à mulher, e, consequentemente reduzir as proporções, ainda persistentes de altas taxas de morbiletalidade materna e fetal. ${ }^{17}$

Em verdade tratava-se de reconhecimentos de recursos tecnológicos adquiridos, estruturados, institucionalizados, além de legitimados ${ }^{18} \mathrm{em}$ prol da saúde materno-infantil. ${ }^{17}$ Constituiu-se em instrumento de fomento para evitar crises, que pudessem tornar a assistência obstétrica menos eficiente. ${ }^{19}$

Com esta tentativa de avanço incrementou-se a atenção pré-natal, e, também, na morbiletalidade materna e fetal, porém, persistindo a não identificação correta das assistências efetivas e cientificamente comprovadas, além de estar sendo relevante contribuição, para dispersar os recursos financeiros destinados à saúde da mulher brasileira. ${ }^{18}$ Além de propiciarem também, elevado ônus econômico para as famílias associados a estes procedimentos. ${ }^{20-22}$

Por certo deva-se reconhecer crédito à contribuição do parto cesáreo nas resoluções obstétricas. 23. Em determinadas ocasiões, também, reconhece-se que o parto cesáreo eletivo, represente procedimento relevante com a finalidade de se poder evitar desnecessariamente, a cesárea em indicações de emergência. ${ }^{24}$

Considerem-se, também, evidências já conhecidas que a elevação das taxas de partos cirúrgicos, não se acompanha em resultados similares de redução sistemática e contínua da morbilidade maternofetal. ${ }^{23}$ A elevação das taxas mundiais de partos cesáreas assumiu tal monta, que a Organização Mundial de Saúde interveio, passando a considerar taxas ao redor de até $15 \%$, além de sugerir a 
implantação da Classificação de Robson, para ser utilizada como parâmetro mundial, para as indicações de partos cesáreas. ${ }^{25,26}$

A mulher brasileira quer adolescente ou adulta demonstra na atualidade sinais de conhecimentos, de que os altos índices de partos cesáreos têm sido desnecessários, estando elas, na verdade, representando como se fora um verdadeiro bem de consumo, utilizado a basto na prática cotidiana na assistência ao parto. ${ }^{27}$

$\mathrm{Na}$ contramão destes fatos não se justifica o crescente número de partos cesáreos, creditado à elevação de demandas judiciais por maus resultados maternos e neonatais, ${ }^{28} \mathrm{e}$, tampouco, à não preocupação das escolas médicas na boa formação dos tocólogos em capacitá-los adequadamente no exercício da tocurgia. ${ }^{29}$ A possível introdução de novas tecnologias assistenciais atuais, podem não representar ou proporcionar as experiências de partos mais satisfatórios para a comunidade feminina de nosso país. ${ }^{30}$

$\mathrm{Na}$ presente pesquisa observou-se a maior e significante proporção de mulheres que ao adentrarem na maternidade, manifestaram o desejo de poderem ter parto vaginal. Seria em verdade o reconhecimento de sua capacidade de ser mãe. Aquelas que o conseguiram, sentiram-se mais recompensadas, com o encontro com seu recém-nascido, a acolhida do ambiente hospitalar, com médicos e enfermagem. O estudo atual, em verdade, teve o mérito de observar a importância da atuação educativa durante a assistência pré-natal, tornando a revelar explicitamente, o papel ativo da mulher durante o processo da parturição. ${ }^{31}$

Em contraponto foi significante, que apesar de ser preferido o parto vaginal, eliminando provável incapacidade para a execução do mesmo, foi mais doloroso e demorado do que quando submetidas às cesáreas, apesar do receio e o medo de morte que elas tinham em relação aos procedimentos anestésicos dos partos cirúrgicos. Os resultados serviram como instrumento útil e relevante, configurando a necessidade de conforto e satisfação para as parturientes, desvelando perspectivas de assistência obstétrica humanizada, como é preconizada pela Organização Mundial de Saúde. ${ }^{32}$

Dentro destas estratégias e suporte de melhora do desconforto doloroso, como vários estudos a basto têm demonstrado, poderia ser a tentativa de implantação de analgesia, durante a evolução e assistência ao parto. ${ }^{33}$

A assistência adequada ao parto deverá consistir em atenções dirigidas a sua evolução, devolvendo a autoconfiança à mulher para tal sem instrumentalizações desnecessárias, não interferindo em sua fisiologia ${ }^{34}$, reconhecendo ser o mesmo, evento familiar e social de maior relevância. ${ }^{5}$

Espera-se que esta pesquisa tenha sido continuação de sentinela, na qualidade e melhora da assistência obstétrica nacional, e, em seu final tenha trazido subsídios às demais populações. 


\section{CONCLUSÕES}

1. Por ser opção pessoal, recuperação pós-parto mais rápida, sensação de melhor encontro com seu recém-nascido e receio de acidentes anestésicos representaram a significativa preferência das puérperas pelo parto vaginal.

2. As experiências negativas para o parto vaginal, com significância estatística foi a sua duração, o desconforto doloroso e provável incapacidade para em sua execução.

3. Os impactos negativos ao parto vaginal seriam minimizados com atenções assistenciais adequadas devolvendo a autoconfiança às pacientes, além da utilização da analgesia intraparto.

4. Apesar de significantemente menor em preferência, o parto cesáreo foi avaliado positivamente, ao receio da experiência dolorosa da parturição, e por sua rapidez resolutiva.

5. A cesárea. foi significante e negativamente procedimento contra si, a possibilidade de acidente anestésico, 


\section{REFERÊNCIAS}

1. Gozzo TO, Fustinoni SM, Barbieri M, Roher WM, Freitas IA. Sexualidade feminina: compreendendo seu significado. Rev Latino-am Enferm. 2000; 8: 84-90.

2. Gomes WA, Costa COM, Sobrinho CLN, Santos CAST, Bacelar EB. Nível de informação sobre adolescência, puberdade e sexualidade entre adolescentes. J Pediatr (Rio de Janeiro). 2002; 78: 52834.

3. Carvacho IE, Silva JLP, Mello MB. Conhecimento de adolescentes grávidas sobre anatomia e fisiologia da reprodução. Rev Assoc Med Bras. 2008; 54: 29-35.

4. Ministério da Saúde (BR). Secretaria de Políticas de Saúde. Área Técnica da Saúde da Mulher. Parto, aborto e puerpério: assistência humanizada à mulher. Brasília (DF). Direitos reprodutivos, saúde materna e perinatal. 2001. p. 11-6.

5. Bezerra MGA, Cardoso MVLML. Fatores interferentes no comportamento das parturientes: enfoque na etnoenfermagem. Rev Bras Enferm. 2005; 58: 698-702.

6. Ministério da Saúde (BR). Secretaria de Políticas de Saúde. Área Técnica da Saúde da Mulher. Parto, aborto e puerpério: assistência humanizada à mulher. Brasília (DF). Atenção institucional ao parto. 2001. p. 17-25.

7. Tedesco RP, Maia Filho NL, Mathias L, Benez AL, Castro VCL, Bourroul GM, Reis FI. Fatores Determinantes para as Expectativas de Primigestas acerca da Via de Parto. Rev Bras Ginecol Obstet. 2004; 26: 791-8.

8. Ministério da Saúde (BR). Secretaria de Políticas de Saúde. Área Técnica da Saúde da Mulher. Parto, aborto e puerpério: assistência humanizada à mulher. Brasília (DF). Avaliação crítica do tipo de parto. 2001. p. 32-7.

9. Código de Nuremberg e Declaração de Helsinki; [acesso em 10 out. de 2020]. Disponível em: http://www.efdeportes.com/efd183/codigo-de-nuremberg-e-declaracao-de-helsinki.htm.

10. Resolução 466/12- Conselho Nacional de Saúde; [acesso em 10 de out. 2020]. Disponível em: http://conselho.saude.gov.br/resolucoes/2012/Reso466.pdf

11. Junqueira SM, Tsunechiro MA. Sentimentos, percepções e necessidades da parturiente na sala de parto. São Paulo: Escola de Enfermagem USP; 1988; 22: 148-60

12. Lopes RCS, Donelli TS, Lima CM, Piccinini CA. O Antes e o Depois: Expectativas e Experiências de Mães sobre o Parto. Psicologia: Reflexão e Crítica. 2005; 18: 247-54.

13. Likert R. A Techinique for the Measurement of Attitudes. Arch Psyc 1932; 140: 1-55

14. Siegel S, Castelani Jr NJ. Estatística não paramétrica para ciências do comportamento. $2^{a}$ ed. Porto Alegre. Artmed; 2006. p. 448.

15. Landis JR, Kock GG. The measurement of observer agreement for contrasts among multinomial populations. Biometrics. 1977; 33: 159-74. 
16. Conover WJ. Practical Nonparametric Statistics, $3^{\text {rd }}$ ed., New York: John Woley \& Sons, 1999.

17. Serruya SJ, Cecatti JG, Lago TG. O Programa de Humanização no Pré-natal e Nascimento do Ministério da Saúde no Brasil: resultados iniciais. Cad Saúde Pública (Rio de Janeiro). 2004; 20: 1281 9.

18. Santos AM. Abordagem social do conhecimento. Lat Am J Develop. 2020; 4: 129-35.

19. Panisset KSP, Santos Jr JDO, Pereira BB, Cunha AJJA. Sazonalidade dos nascimentos no Estado do Rio de Janeiro: 1995-2005. Lat Am J Develop 2021: 6: 671-88.

20. Silva JLP, Cecatti JG, Serruya SJ. A qualidade do pré-natal no Brasil. Rev Bras Ginecol Obstet. 2005; 27: 103-5.

21. Mylonas I, Friese K. Indications for and risks of elective cesarean section. Dtsch Arztebl Int 2015; 112: 489-95.

22. Haider MR, Rahman MM, Moiniddin M, Rahman AE, Shakil A. Khan MM. Ever - increasing Caesarean section and its economic burden in Bangladesh. 2018; 13 (12): e0208623.

23. Silva SALC, Moraes Filho OB, Costa CFF. Análise dos fatores de risco anteparto para ocorrência de cesárea. Rev Bras Ginecol Obstet. 2005; 27: 189-96.

24. Nomura RMY, Alves EA, Zugaib M. Complicações maternas associadas ao tipo de parto em hospital universitário. Rev Saúde Pública. 2004; 38: 9-15.

25. FIGO Working Group on Challenges in Care of Mothers and Infants during Labour and Delivery. Best practice advice on the 10-Group Classification System for cesarean deliveries. Int J Gynecol Obstet 2016; 135: 232-3.

26. Jogia PD, Lodhiya KK. Analysis of cesarean sections according to modified Robson's ten group classification system at a tertiary care center in Western India. Int J Reprod Contracept Obstet Gynecol 2019; 8: 433-9.

27. Benzadeli DS, Tavares BB. Expectativa quanto ao parto e conhecimento do motivo da cesárea: entre puérperas adolescentes e adultas. Rev Elet Enf 2010; 12: 150-7.

28. Maia Filho NL, Mathias L, Hiar J, Pientznauer R, Carvalho PM. Operação cesariana - A propósito de suas indicações. J Bras Ginecol. 1994; 104: 423-6.

29. Faúndes A, Silva JLP. O equilíbrio entre o parto vaginal e cesárea na assistência obstétrica atual. Femina. 1988; 26: 669-73.

30. Costa R, Figueiredo B, Pacheco A, Pais A. Parto: Expectativas, Experiências, Dor e Satisfação. Psicologia, Saúde \& Doenças. 2003; 4: 47-67.

31. Gonçalves AK, Míssio L. Fatores determinantes para as expectativas de gestantes acerca da via de parto. Anais do $7^{\circ}$ ENIC. 2000. 
32. Almeida NAM. A dor do parto normal na perspectiva e vivência de um grupo de mulheres usuárias do Sistema Único de Saúde. Tese. Programa Multi-institucional de Pós-Graduação em Ciências da Saúde. Goiânia. 2009.

33. Novo JLVG, Neme B. Efeitos da manobra de Kristeller e do fórcipe de alívio sobre o sistema vascular fetal. Estudo comparativo pelo exame de fundo de olho. Ginec e Obstet Bras 1978; 1: 173-84.

34. Petruce LFF, Oliveira LR, Oliveira VR, Oliveira SR. Humanização no atendimento ao parto baseado em evidências. Femina. 2021; 45: 212-22. 